Supporting Information for

\title{
Ring-Opening Hydration of Epoxides into Diols with a Low Water- Epoxide Proportion Catalyzed by a Fe-Incorporated Octahedra-Based Molecular Sieve
}

Duo Wang, ${ }^{a \dagger}$ Yao Yang, ${ }^{a \dagger}$ Fenhua Zhang, ${ }^{a}$ Pengfei Jiang, ${ }^{a}$ Wenliang Gao, ${ }^{a *}$ Rihong Cong, ${ }^{a b}$ Tao Yang $^{a b^{*}}$

${ }^{a}$ College of Chemistry and Chemical Engineering, Chongqing University, Chongqing 401331, People's Republic of China

${ }^{\mathrm{b}}$ Chongqing Key Laboratory of Theoretical and Computational Chemistry, Chongqing University, Chongqing 401331, People’s Republic of China

$\dagger$ D. Wang and Y. Yang contributed equally.

* Corresponding authors: gaowl@cqu.edu.cn; taoyang@cqu.edu.cn 


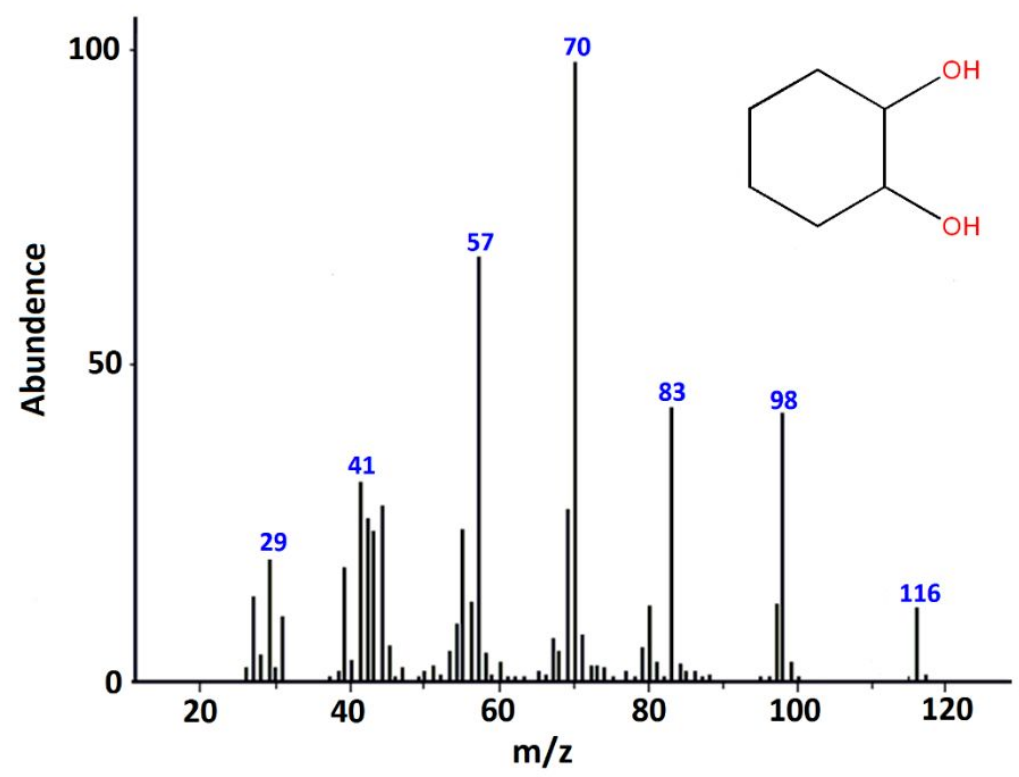

Figure S1. Mass spectrum of the product, which is identical to 1,2-cyclohexanediol.

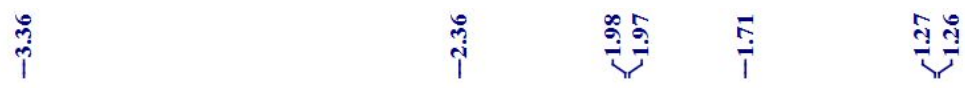

${ }^{1} \mathrm{H}$ NMR $\left(600 \mathrm{MHz}, \mathrm{cdcl}_{3}\right) \delta 3.36(\mathrm{~s}, 1 \mathrm{H}), 2.36(\mathrm{~s}, 1 \mathrm{H}), 1.98(\mathrm{~d}, J=5.5$

$\mathrm{Hz}, 1 \mathrm{H}), 1.71(\mathrm{~s}, 1 \mathrm{H}), 1.35-1.19(\mathrm{~m}, 2 \mathrm{H})$.
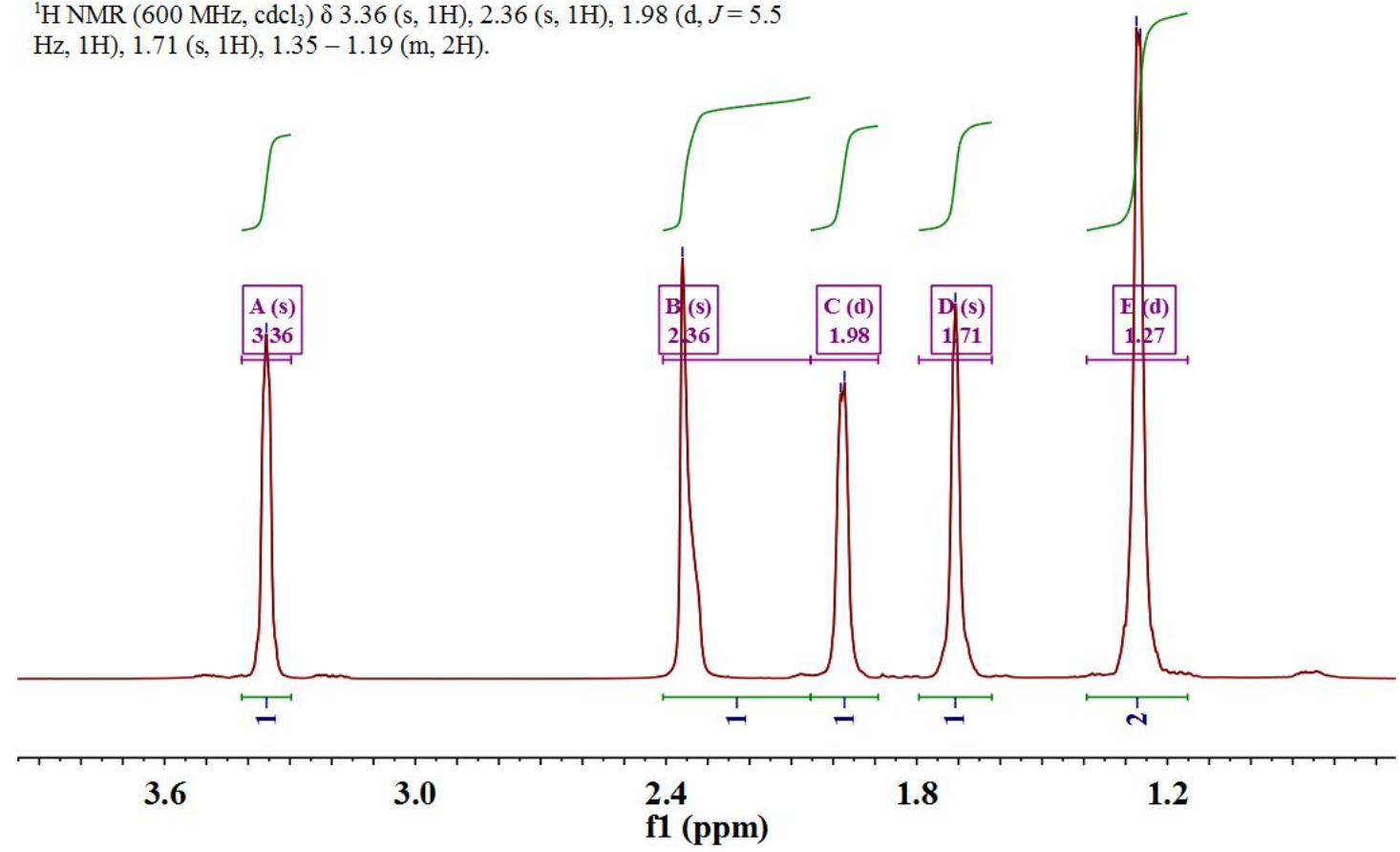

Figure S2. ${ }^{1} \mathrm{H}$ NMR spectrum of the reaction product (1,2-cyclohexanediol). 


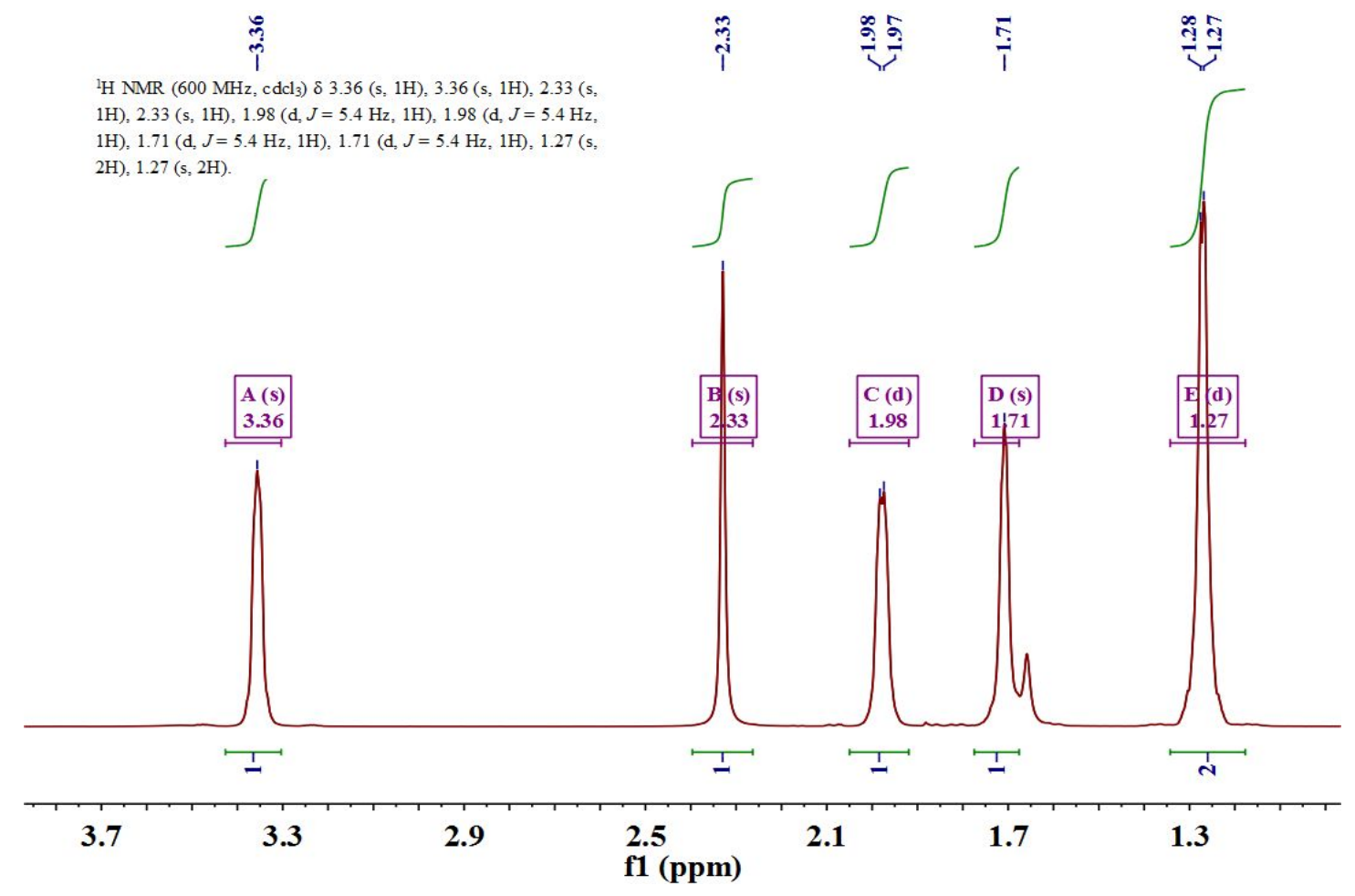

Figure S3. ${ }^{1} \mathrm{H}$ NMR spectrum of the commercial trans-1,2-cyclohexanediol.

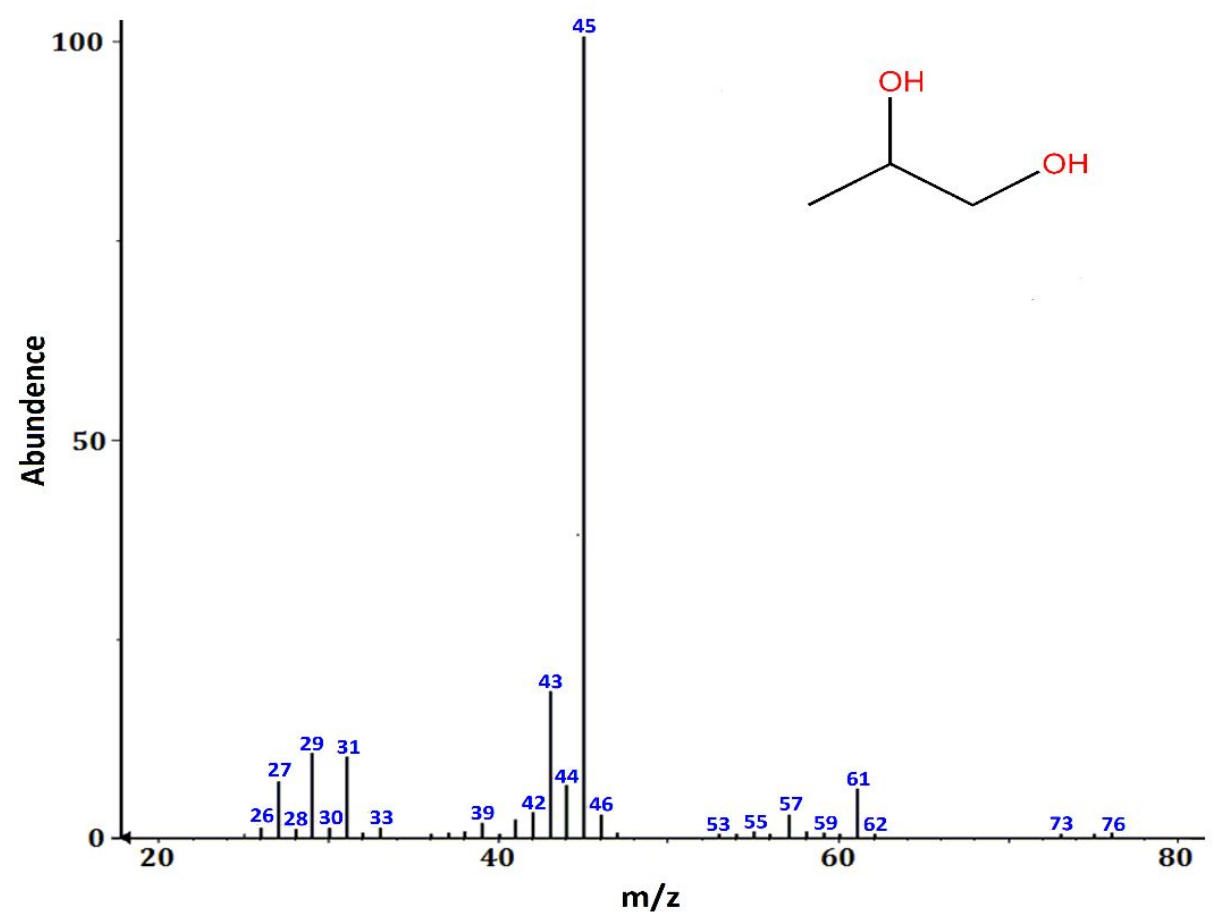

Figure S4. Mass spectrum of the reaction product (1,2-propanediol) 


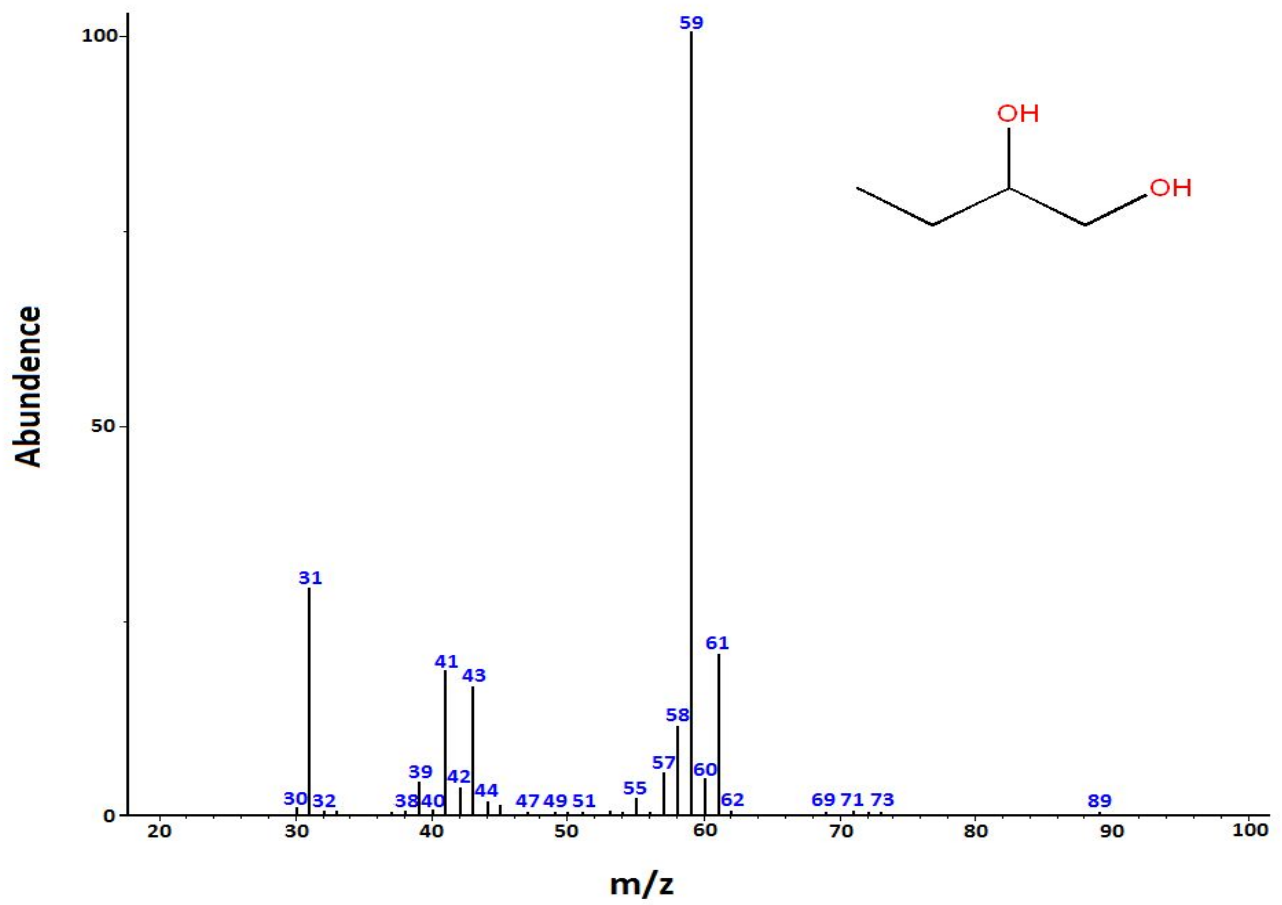

Figure S5. Mass spectrum of the reaction product (1,2-butanediol).

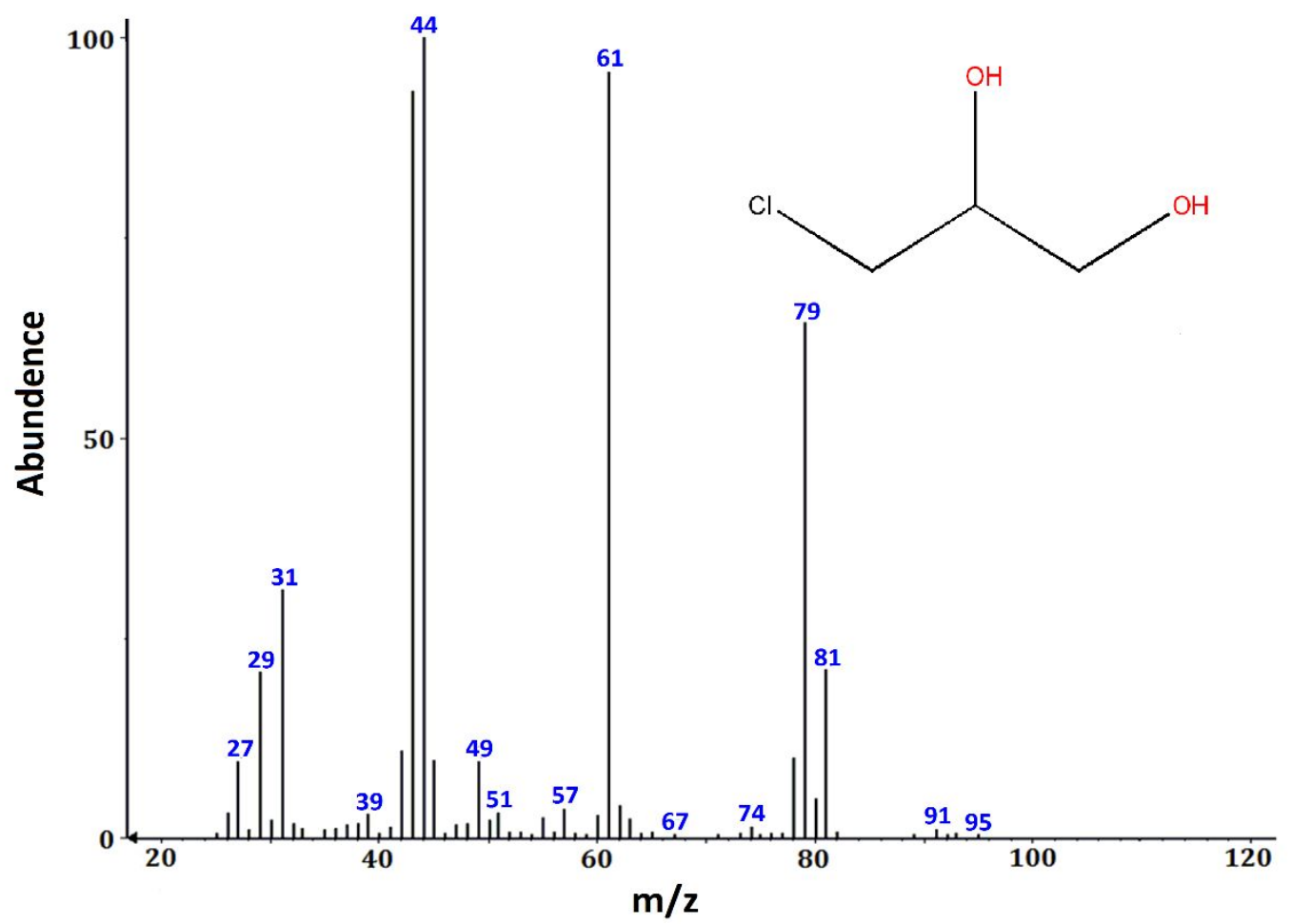

Figure S6. Mass spectrum of the reaction product (3-chloro-1,2-propanediol). 


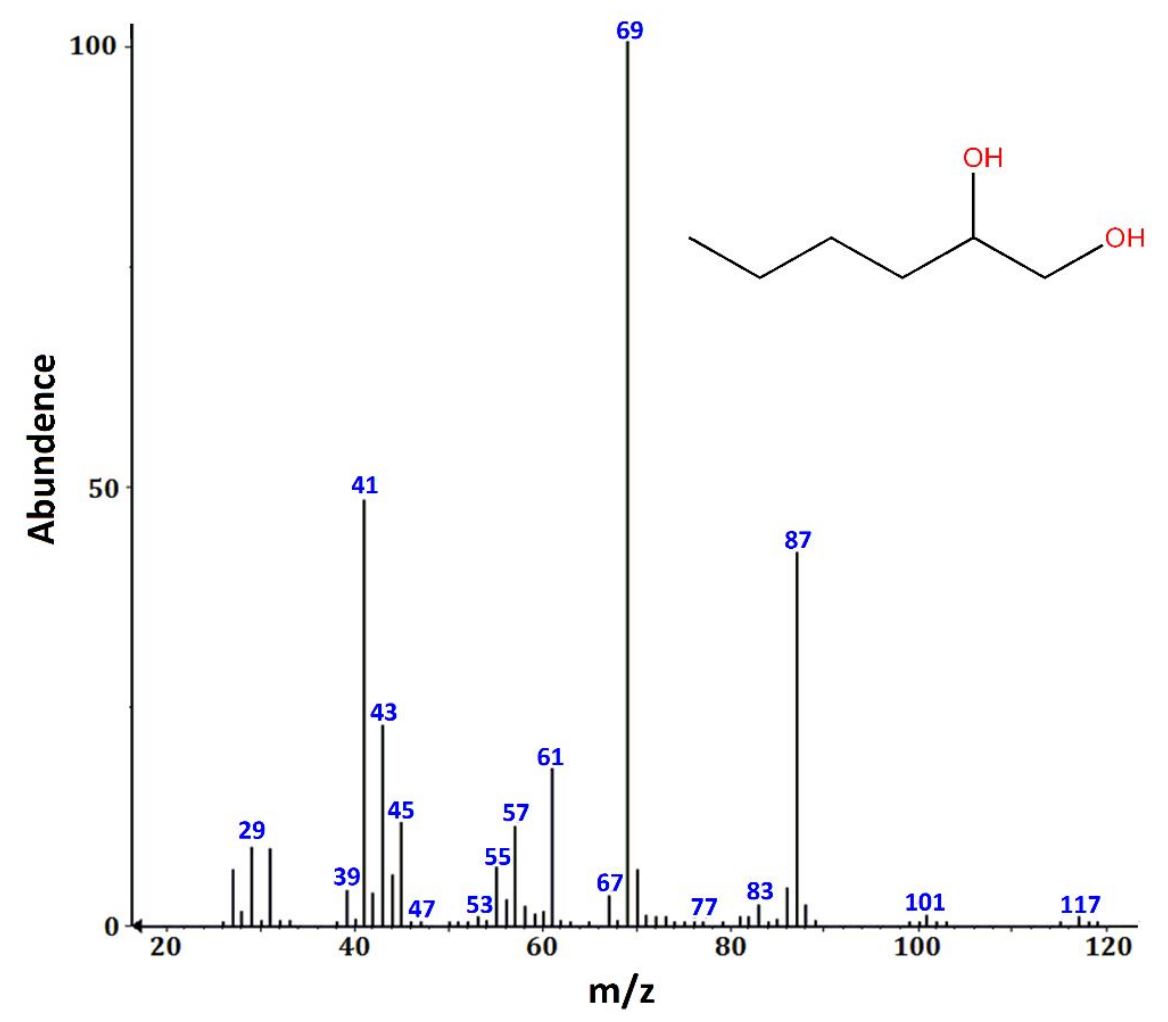

Figure S7. Mass spectrum of the reaction product (1,2-hexanediol).
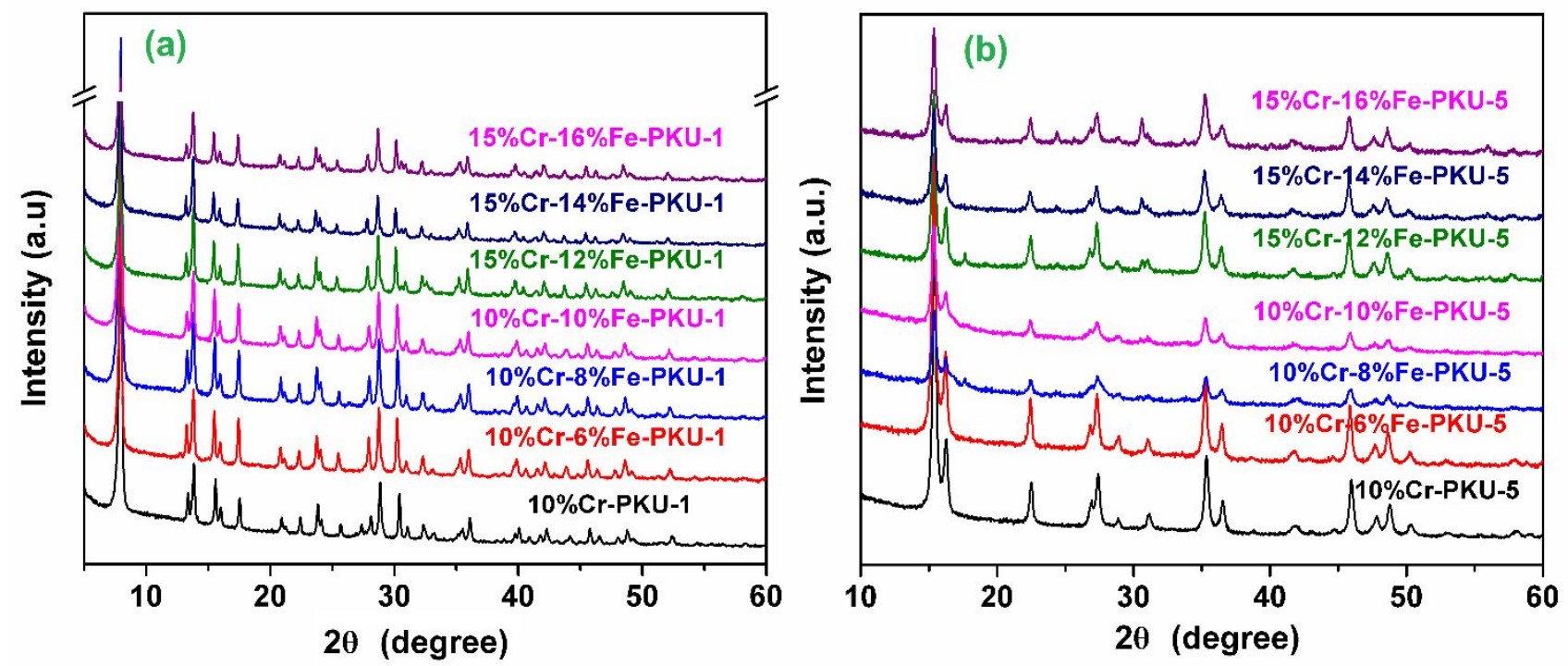

Figure S8. XRD patterns of Cr-Fe-PKU-1 and Cr-Fe-PKU-1 catalysts. 

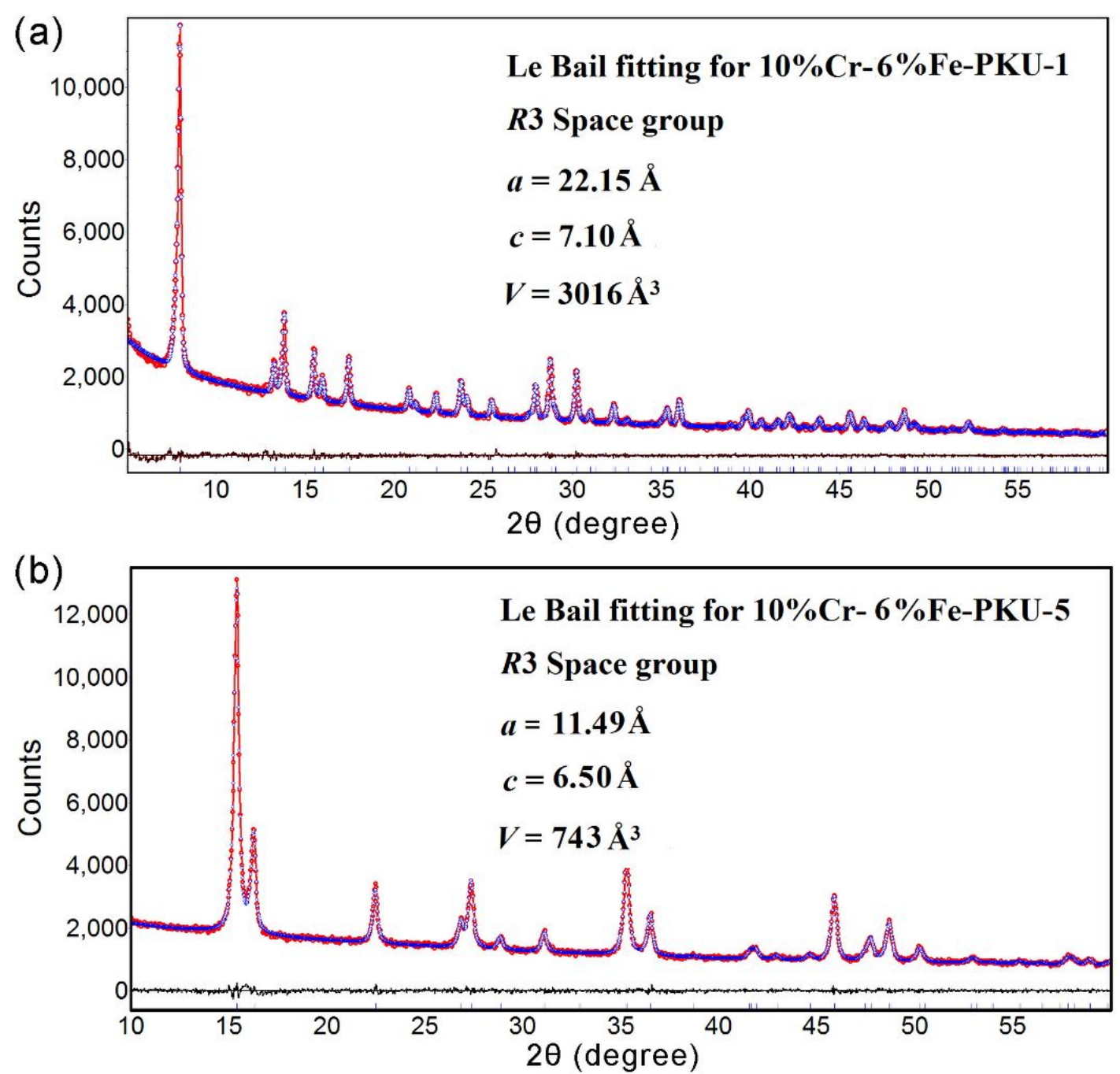

Figure S9. The Le Bail fitting of powder XRD patterns for $10 \% \mathrm{Cr}-6 \% \mathrm{Fe}-\mathrm{PKU}-1$ (a) and $10 \% \mathrm{Cr}-6 \%$ Fe-PKU-5 (b), blue circles and red solid line represent the observed and the calculated data; the marks below are the expected reflections. 